\title{
Gastrointestinal blood flow velocity after the first feed
}

\author{
G Gladman, D G Sims, M L Chiswick
}

\begin{abstract}
Twenty preterm infants (gestational age range 27-36 weeks) who had never been fed enterally were studied. Doppler indices of flow velocity from the superior mesenteric and coeliac arteries were measured immediately before, and at postprandial time intervals up to 60 minutes after, a first feed of $1 \mathrm{ml}$ of milk given through a nasogastric tube. There were no significant differences between the preprandial and postprandial values of blood pressure, heart rate, oxygen and carbon dioxide tensions, and $\mathrm{pH}$.

The median peak systolic velocity (PSV) $(55.9 \mathrm{~cm} / \mathrm{sec})$ and time average mean velocity (TAV) $(10.6 \mathrm{~cm} / \mathrm{sec})$ before the feed at the superior mesenteric artery rose progressively and significantly to postprandial peaks of 88.5 $\mathrm{cm} / \mathrm{sec}$ and $20.5 \mathrm{~cm} / \mathrm{sec}$ at 45 minutes, respectively. Significant postprandial rises in the median PSV and TAV values at the coeliac artery were also observed, with a peak at 45 minutes. There were no significant correlations between blood flow velocity indices (preprandial or postprandial) and gestational age, postnatal age, conceptional age, or birth weight.

We conclude that the first exposure of preterm babies to a small volume of milk feed is associated with postprandial changes in blood flow velocity consistent with an increase in blood flow in the superior mesenteric and coeliac arteries, although the mechanism of this response is uncertain.
\end{abstract}

It is the practice in many neonatal units to delay the introduction of enteral feeding in preterm infants if they are unwell. Basal plasma concentrations of certain gut hormones, ${ }^{2}$ postprandial surges in their plasma concentrations, ${ }^{34}$ and the postprandial motor response of the small intestine ${ }^{5}$ in preterm infants are to some extent dependent on the magnitude of previous exposure of the gut to milk feeds.

Doppler ultrasound measurements of flow velocity in the superior mesenteric artery have been reported in adults ${ }^{6}$ and more recently in preterm infants. ${ }^{8}$ To our knowledge there have been no reports of postprandial flow velocity in abdominal vessels in response to the introduction of a uniform, small bolus of milk in preterm infants who have not previously received enteral feeds. Recently Leidig reported postprandial changes in flow velocity from the superior mesenteric arteries of preterm infants, but most of these infants had already been established on enteral feeds and their postpran- dial flow velocity was measured after varying volumes of bolus feed. ${ }^{8}$

In our neonatal unit it is usual to start milk feeding in very preterm babies with bolus volumes as small as $1 \mathrm{ml}$ at hourly intervals. It is not uncommon for the nurses to observe self correcting hypoxaemic episodes (on transcutaneous monitoring), with or without bradycardias and apnoeas, within a few hours of the start of feeding that resolve when feeding is temporarily discontinued. It was not the purpose of this study to explore the relationship of these episodes to postprandial changes in flow velocity in gastrointestinal arteries. Instead, we wished to determine whether in very preterm babies of different postnatal ages, the first enteral feed of a very small bolus of milk was associated with postprandial changes in Doppler flow velocity measured at the superior mesenteric and coeliac arteries.

\section{Patients and methods}

We studied 20 preterm infants (seven girls and 13 boys) whose weight and age characteristics are shown in table 1. Although some had previously been treated for respiratory distress none had ever shown signs or symptoms of intestinal disease and all were apparently healthy at the time of the study with no clinical signs of patent ductus arteriosus. Umbilical catheters had been removed at least 24 hours before the start of the study. None of the infants had previously received any food enterally.

A nasogastric feeding tube (deadspace 0.25 $\mathrm{ml}$ ) was passed and after a 30 minute period of stabilisation, baseline Doppler blood flow velocities in the superior mesenteric and coeliac arteries were measured. Each baby was then 'fed' with $1.25 \mathrm{ml}$ of milk introduced through the nasogastric tube. Sixteen babies received a modified cows' milk formula, and the remainder expressed breast milk, according to the mother's preference.

Blood flow velocity measurements from both arteries were repeated at $5,15,30,45$, and 60 minutes after this first feed. At the time of each Doppler measurement the heart rate and blood pressure (Dinamap) were recorded for each baby. In five of the 20 infants peripheral arterial

Table 1 Median (range) ages and birth weights of the 20 babies

\begin{tabular}{lc}
\hline Gestational age (weeks) & $29(27-36)$ \\
Birth weight (g) & $1248(910-2480)$ \\
Postnatal age (days) & $7(1-33)$ \\
Conceptional age (weeks) & $31(28-37)$ \\
\hline
\end{tabular}




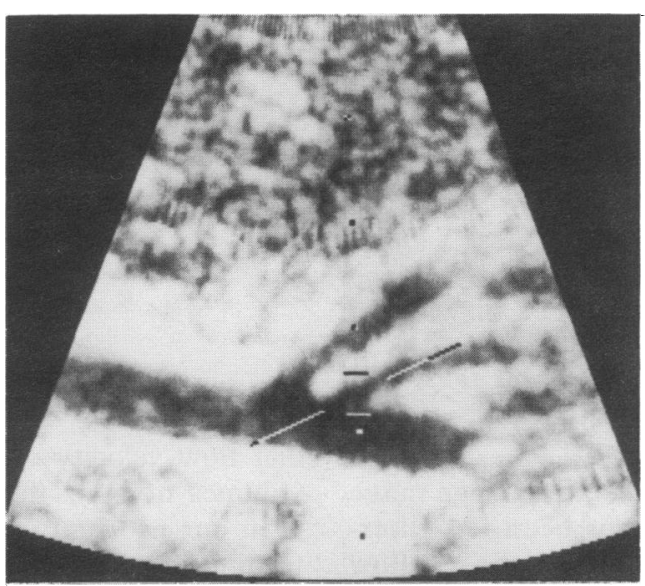

Figure 1 Longitudinal abdominal ultrasound scan showing the coeliac and superior mesenteric arteries as they arise from the aorta. Doppler sample gate is positioned at the origin of the superior mesenteric artery.

catheters had been left in position and in these babies we measured oxygen and carbon dioxide tensions and $\mathrm{pH}$ before, and 60 minutes after, the feed.
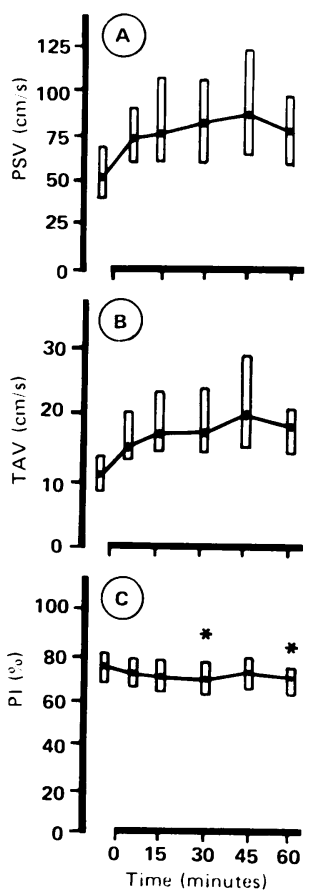

Figure 2 Median and interquartile ranges of: (A) peak systolic velocity (PSV); (B) time average mean velocity $(T A V)$; and (C) Pourcelot resistance index $(P I)$ measured from the superior mesenteric artery, before the feed ( 0 minutes) and at different postprandial times. At each postprandial time, the median PSV and TAV were significantly higher than before the feed $(p<0.01) ;{ }^{*} p<0.05$ compared with before the feed.
MEASUREMENTS OF BLOOD FLOW VELOCITY

Recordings were made with a duplex ultrasound scanner (Acuson 128) equipped with a S519 probe, which has a pulse echo frequency of 5.0 $\mathrm{MHz}$ and a Doppler frequency of $5 \cdot 0 \mathrm{MHz}$. The high pass wall filter was set at $125 \mathrm{~Hz}$ and the ultrasound intensity was kept to a minimum compatible with the collection of an adequate signal (always less than $150 \mathrm{~mW} / \mathrm{cm}^{2}$ spatial peak, temporal average).

The superior mesenteric and coeliac arteries were visualised through longitudinal abdominal scans using real time imaging, which allowed placement of the Doppler sample (gate size $3 \mathrm{~mm}$ ) roughly $5 \mathrm{~mm}$ distal to the origin of each artery from the aorta (fig 1). The system allowed for an automatic 'angle correction' to $0^{\circ}$ in the velocity calculations.

The maximal Doppler signal was obtained both visibly and audibly. The system permitted up to 20 consecutive cardiac cycles to be captured and analysed by fast fourier transformation. In practice, the quality of the Doppler signal could rarely be maintained to capture this number of consecutive cycles. On average we were able to analyse $\mathbf{1 0}$ consecutive cycles, and in no baby did we analyse less than five cycles.

The built in microcomputer permitted immediate analysis of the frozen velocity waveform, and the indices chosen for the study were the peak systolic velocity (PSV), the time average mean velocity (TAV) and the Pourcelot resistance index (PI), which was defined as (S-D) divided by $S$, where $(S)$ is the maximum systolic frequency shift, and (D) the minimum diastolic frequency shift. This is believed to be an index of vascular resistance downstream from the point of Doppler insonation.

\section{REPEATABILITY OF METHOD AND STATISTICAL} METHODS

We assessed the repeatability of the method by making 50 paired blood flow velocity record- ings, five minutes apart, from both the superior mesenteric and coeliac arteries in healthy, sleeping, preterm babies who had never been fed orally. The ultrasound probe was removed between examinations. From each artery, we calculated the mean value of paired measurements of PSV, TAV, and PI $(n=100)$ and the $S D$ of the difference between pairs $(n=50)$. We also calculated the corresponding coefficient of variation of these measurements with 0.95 probability. In the study analyses, the significance of differences between preprandial and postprandial measurements was tested by the Wilcoxon signed rank test (two tailed level of significance is quoted). The significance of the correlation between the various ultrasound measurements and gestational age, postnatal age, conceptional age, and birth weight was tested by Kendall's rank correlation.

\section{Results}

In our assessment of repeatability the mean values of measurements of PSV, TAV, and PI from the superior mesenteric artery (in each case $n=100$ ), and the SD of the difference between pairs, were $62.5(4.6 \mathrm{~cm} / \mathrm{sec}) ; 18.0(1 \cdot 3$ $\mathrm{cm} / \mathrm{sec})$, and $69 \cdot 0(3 \cdot 1 \%)$. The corresponding results expressed as coefficients of variation with 0.95 probability were $15 \%, 14 \%$, and $9 \%$, respectively. Similar results were obtained from the coeliac artery: mean values (SD of difference between pairs) for PSV, TAV, and PI were $67 \cdot 3(4 \cdot 3 \mathrm{~cm} / \mathrm{sec}) ; 19 \cdot 0(1 \cdot 2 \mathrm{~cm} / \mathrm{sec})$, and 66 $(3.4 \%)$, with corresponding coefficients of variation with 0.95 probability of $13 \%, 13 \%$, and $10 \%$.

The median PSV and TAV from the superior mesenteric artery before the feed were $55.9 \mathrm{~cm} /$ sec and $10.6 \mathrm{~cm} / \mathrm{sec}$, respectively, and rose to a postprandial peak of $88.5 \mathrm{~cm} / \mathrm{sec}$ and $20.5 \mathrm{~cm} /$ sec at 45 minutes (fig 2). Similarly in the coeliac artery PSV and TAV rose from median values before the feed of $61.5 \mathrm{~cm} / \mathrm{sec}$ and $18.3 \mathrm{~cm} / \mathrm{sec}$, respectively, to postprandial peaks of $108.0 \mathrm{~cm} /$ sec and $31.5 \mathrm{~cm} / \mathrm{sec}$ at 45 minutes (fig 3). At each postprandial interval the median PSV and TAV from each artery were significantly higher than before the feed $(p<0.01)$. In individual infants the blood flow velocity peaked at different times. Table 2 shows the time interval after the feed when babies had their maximal increases in PSV and TAV measured in the superior mesenteric artery. In most infants the peak velocity occurred at 45 minutes after the feed. Similar changes were observed from the coeliac artery.

The median PI before the feed in the superior mesenteric artery was $76 \%$, and this fell postprandially to a significantly lower value at 30 minutes $(69 \cdot 5 \%)$ and 60 minutes $(71 \%)$ $(\mathrm{p}<0.05)$ (fig 2$)$. We did not observe any significant change in PI measured from the coeliac artery (fig 3).

There was no correlation between blood flow velocity indices (preprandial or postprandial) on the one hand, and gestational age, postnatal age, conceptional age, or birth weight on the other. There were no significant differences between preprandial and postprandial values of blood 
Table 2 Postprandial timing of maximum peak systolic velocity and average mean velocity measured in superior mesenteric artery in 20 babies

\begin{tabular}{lcl}
\hline $\begin{array}{l}\text { Postprandial time } \\
\text { (mins) }\end{array}$ & $\begin{array}{l}\text { Maximum peak } \\
\text { systolic velocity }\end{array}$ & $\begin{array}{l}\text { Time average } \\
\text { mean velocity }\end{array}$ \\
\hline 5 & 2 & 0 \\
15 & 2 & 1 \\
30 & 4 & 7 \\
45 & 11 & 9 \\
60 & 1 & 3 \\
\hline
\end{tabular}

pressure, heart rate, or oxygen and carbon dioxide tensions and $\mathrm{pH}$.

In only four of the 20 infants was feeding subsequently stopped, and in all but one of these the decision was made because of episodic bradycardia. We did not observe any difference in the blood flow velocity patterns between infants in whom we subsequently withdrew feeds and those who continued to feed. It was not the purpose of this study to compare the influence of different milks on blood flow velocity, but the four infants who received expressed breast milk had similar trends in their flow velocities as the infants who received formula milk.
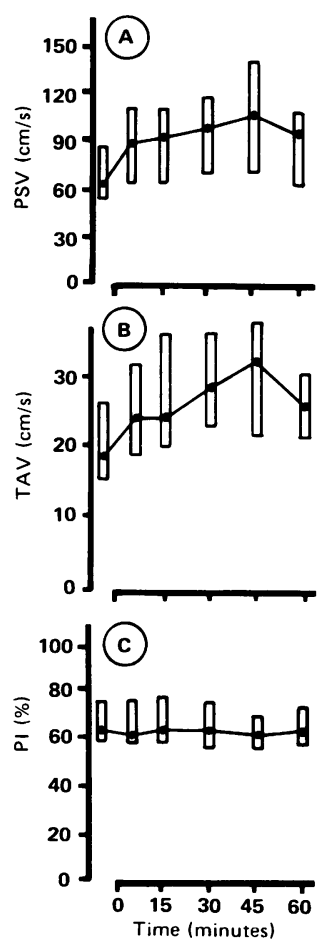

Figure 3 Median and interquartile ranges of: (A) peak systolic velocity (PSV); (B) time average mean velocity (TAV); (C) Pourcelot resistance index $(P I)$ measured from the coeliac artery, before the feed ( 0 minutes) and at different postprandial times. At each postprandial time, the median PSV and TAV were significantly higher than before the feed $(p<0.01)$.
Leidig measured changes in PSV, TAV, and PI from the superior mesenteric artery in pre- term babies, but most of these infants had already been exposed to milk feeds and measurements were made after varying volumes of bolus feeds. ${ }^{8} \mathrm{He}$ also observed that PSV and TAV rose by 15 minutes after the feed and peaked at 45 minutes. These changes were associated with a fall in PI. It was further observed that 'basal' flow velocities were higher in infants who had received larger volumes of milk. All infants had started feeding within 24 hours of birth with volumes according to postnatal age. It was, therefore, not possible to know whether postnatal age (or conceptional age) or previous 'milk loading' influenced basal flow velocities. As none of the infants in our study had previously received milk, basal flow velocity measurements were independent of exposure to feeds. None the less, we observed no correlation between any of the Doppler indices of flow from either artery with postnatal or conceptional age.

The control of intestinal blood flow in adults is complex and is determined by local regulatory mechanisms that are independent of neural control (metabolic and myogenic); extrinsic regulation (autonomic nervous system); and by circulating vasoactive substances. ${ }^{10}$ Virtually nothing is known about the development of these regulatory mechanisms in fetal life and early infancy. We consider it unlikely that the increase in flow velocities observed after the small volume feed was related to an increase in small intestine motor activity, because Bisset $\boldsymbol{e t}$ al observed disruption of fasting motor activity only after large bolus feeds in infants who had already been well established on oral feeds. ${ }^{5}$

It is important to note that similar changes were observed when measurements were made from both the coeliac axis and the superior mesenteric artery, consistent with there being an increase in blood flow to the stomach as well as the small intestine. Qamar et al, using a duplex scanning system, showed that there was an immediate increase in coeliac axis flow velocity that persisted for 10 minutes and returned to resting levels by 30 minutes in adults after the ingestion of a liquid meal. ${ }^{11}$ In dogs a small increase in coeliac axis blood flow was observed after intragastric placement of food. ${ }^{12}$

In our study the relationship between coeliac axis flow and superior mesenteric flow is unclear, although the time course of the rise after the $1 \mathrm{ml}$ feed was similar, peaking at 45 minutes. We did not observe a significant effect on PI measured from the coeliac axis after the feed, although PI fell when measured from the superior mesenteric artery. If it is true that PI is an index of 'downstream' resistance, then a Doppler sample from the coeliac axis will reflect splenic and hepatic vascular resistance as well as the resistance of the gastric arterial bed. In this respect it is of interest that radioisotope imaging after a bolus injection of technetium in adults showed that there was a reduction in splenic and hepatic arterial flow during digestion. ${ }^{13}$

The fact that a rise in flow velocity was observed as rapidly as five minutes after the introduction of the feed suggests that there is a flow regulatory mechanism operating - at least initially-in the stomach. It is possible that 
mechanical stimulation of the gastric mucosa by the milk bolus triggered the changes in blood flow velocity through a local reflex. Certainly mechanical stimulation of a denervated jejunal segment causes an increase in blood flow, a response which is probably not limited to the mucosa and which is blocked by 5-hydroxytryptamine. ${ }^{14}$ If mechanical stimulation of the gastric mucosa was in part responsible for triggering the changes in flow velocity then there is no reason to suppose that the feeding tube itself was responsible as this was left in position undisturbed throughout the study.

A number of gastrointestinal and pancreatic polypeptide hormones such as secretin, cholecystokinin, and glucagon have been shown to increase intestinal blood flow in adults, ${ }^{10}$ but the diversity of the experimental conditions is such that it is difficult to draw conclusions about a physiological role for any of these hormones in the regulation of intestinal or gastric blood flow. Certainly in preterm infants, following the first feed of human milk, plasma glucagon concentration did not rise. ${ }^{3}$

The synthetic analogue of gastrin, pentagastrin, is a potent intestinal vasodilator, ${ }^{10}$ but little is known about the vasomotor effects of gastrin in a physiological setting. Although term babies have a postprandial rise in plasma gastrin concentration during the hour after the first milk feed, ${ }^{15}$ a postprandial response is not observed in preterm babies until 13 days postnatal age, even though they are already receiving regular milk feeds. ${ }^{4}$ Preterm babies, 6 days old, who had never received milk, had lower basal plasma concentrations of gastrin compared with those of an equivalent age who had been fed. ${ }^{1}$ Fourteen of the 20 infants in our study had their first bolus feed and flow velocity measurements at a postnatal age of 8 days or less. Based on the observations cited above, even if gastrin was a gastrointestinal vasodilator it is unlikely that a postprandial surge in plasma gastrin was implicated in the observed rise in flow velocities.

Whether the changes in flow velocity we have observed in this study are initiated by a stimulus arising in the stomach or beyond the stomach (or both) is unclear. This could probably be clarified by repeating these studies with a small bolus of feed introduced through a transpyloric tube.

1 Lucas A, Adrian TE, Christofides N, Bloom SR, AynsleyGreen A. Plasma motilin, gastrin and enteroglucagon and feeding in the human newborn. Arch Dis Child 1980;55:673-7.

2 Lucas A, Bloom SR, Aynsley-Green A. Postnatal surges in plasma gut hormones in term and preterm infants. Biol Neonate 1982;41:63-7.

3 Lucas A, Bloom SR, Aynsley-Green A. Metabolic and endocrine events at the time of the first feed of milk in preterm and term infants. Arch Dis Child 1987;53:731-6.

4 Lucas A, Bloom SR, Aynsley-Green A. Development of gut hormone responses to feeding in neonates. Arch Dis Child 1980;55:678-82.

5 Bisset WM, Watt J, Rivers RPA, Milla PJ. Postprandial motor response of the small intestine to enteral feeds in preterm infants. Arch Dis Child 1989;64:1356-61.

6 Qamar MI, Read AE, Skidmore R, Evans JM, Wells PNT Transcutaneous Doppler ultrasound measurement of Trerior mesenteric artery blood flow in man. Gut 1986;27:100-5.

7 Qamar MI, Read AE, Skidmore R, Evans JM, Wells PNT. Pulsatility index of superior mesenteric artery blood flow
velocity waveforms. Ultrasound Med Biol 1986;12:773-6.

8 Leidig E. Doppler analysis of superior mesenteric artery blood flow in preterm infants. Arch Dis Child 1989;64: b76-80.

9 Norryd C, Denker H, Lunderquist A, Olin T, Tylen U. Superior mesenteric blood flow during digestion in man. Acta Chir Scand 1975;141:197-202.

10 Granger DN, Richardson PDI, Kvietys PR, Mortillaro NA. Intestinal blood flow. Gastroenterology 1980;78:837-63.

11 Qamar MI, Read AE, Skidmore R, Evans JM, Williamson RCN. Transcutaneous Doppler ultrasound measurement of coeliac axis blood flow in man. Br $\mathcal{J}$ Surg 1985;72:391-3.

12 Chou C, Hsieh C, Yu Y, et al. Localisation of mesenteric hyperemia during digestion in dogs. Am $\mathcal{f}$ Physiol 1976;236:583-9.

13 Walmsley B, Fleming J, Karran S. Hepatic, mesenteric and splenic blood flow after digestion. Gut 1982;23:A886-7.

14 Biber B, Lundgren O, Svanvik J. Studies on the intestinal vasodilation observed after mechanical stimulation of the vasodilation observed after mechanical stimulation of the
mucosa of the gut. Acta Physiol Scand 1971;82:177-90.

15 Von Berger L, Henrichs I, Raptis S, et al. Gastrin concentration in plasma of the neonate at birth and after the first feeding. Pediatrics 1976;58:264-7. 